\title{
An Advanced SOM Algorithm Applied to Handover Management Within LTE
}

\author{
Neil Sinclair, Student Member, IEEE, David Harle, Member, IEEE, Ian A. Glover, Member, IEEE, \\ James Irvine, Senior Member, IEEE, and Robert C. Atkinson, Senior Member, IEEE
}

\begin{abstract}
A novel approach to handover management for Long-Term Evolution (LTE) femtocells is presented. Within LTE, the use of self-organizing networks (SONs) is included as standard, and handover management is one of its use cases. Base stations can autonomously decide whether handover should take place and assign the values of relevant parameters. Due to the limited range of femtocells, handover requires more delicate attention in an indoor scenario to allow for efficient and seamless handover from indoor femtocells to outdoor macrocells. As a result of the complexity of the indoor radio environment, frequent ping-pong handovers between the femtocell and macrocell layers can occur. A novel approach requiring a small amount of additional processing using neural networks is presented. A modified self-organizing map (SOM) is used to allow a femtocell to learn the locations of the indoor environment from where handover requests have occurred and, based on previous experience, decide whether to permit or prohibit these handovers. Once the regions that coincide with unnecessary handovers have been detected, the algorithm can reduce the total number of handovers that occur by up to $70 \%$ while still permitting any necessary handover requests to proceed. By reducing the number of handovers, the overall efficiency of the system will improve as the consequence of a reduction in associated but unnecessary signaling. Using machine learning for this task complies with the plug-and-play functionality required from SONs in LTE systems.
\end{abstract}

Index Terms-Handover, long-term evolution (LTE), neural networks, self-organizing feature maps, self-organizing networks (SON).

\section{INTRODUCTION}

$\mathbf{T}$ HE increasing demand by mobile subscribers for high data rates is a driver for two key aspects of the Long-Term Evolution (LTE) of cellular systems. First, the highly flexible air interface based on orthogonal frequency-division multiple access, which is combined with multiple-input-multiple-output (MIMO) antenna technology, increases bandwidth efficiency compared with that of existing third-generation (3G) systems. Second, LTE-based systems are expected to utilize additional femtocell base stations to meet the demand for high-data-rate

Manuscript received May 15, 2012; revised September 14, 2012 and December 21, 2012; accepted February 9, 2013. Date of publication March 8, 2013; date of current version June 12, 2013. The review of this paper was coordinated by Mr. C. Schmelz.

N. Sinclair, D. Harle, J. Irvine, and R. C. Atkinson are with the Department of Electronic Electrical Engineering, University of Strathclyde, Glasgow G1 1XW, U.K. (e-mail: neil.sinclair@strath.ac.uk; robert.atkinson@strath.ac.uk).

I. A. Glover is with the Department of Engineering and Technology, University of Huddersfield, Huddersfield HD1 3DH, U.K.

Color versions of one or more of the figures in this paper are available online at http://ieeexplore.ieee.org.

Digital Object Identifier 10.1109/TVT.2013.2251922 services. Taking such an approach, users (such as those located indoors) can be assigned to the femtocell layer, thereby freeing capacity at the macrocell layer. In such a context, the femtocell serves an exclusive set of users as defined by a closed subscriber group [1].

Wide-scale deployment of large numbers of base stations has implications for the economic viability of a cellular system. As increasing numbers of base stations are deployed, the manual effort (and, hence, cost) to configure, optimize, and maintain them becomes unsustainable. Furthermore, as the number of base stations increases, the complexity of the system also increases. Recent studies have shown that $70 \%$ of all voice and data traffic derives from users located indoors [2]. However, due to the penetration loss of exterior walls, these users often experience low service quality, which limits them to lower bit-rate connections. LTE's integrated support for femtocell technology directly addresses the issue of penetration loss; femtocells can be deployed indoors and, consequently, provide the required high signal strengths. However, there is a consequent increase in management complexity.

To meet the challenges of increased complexity while driving down both the capital expenditure (CAPEX) and the operational expenditure (OPEX) of their networks, a degree of selfmanagement is proposed for LTE-based systems, which can be achieved using self-organizing networks (SONs) [3]. SONs offer plug-and-play functionality for network elements: Base stations (eNodeBs and HeNodeBs) can be deployed and will automatically configure their radio parameters with minimum human interaction. Within $3 \mathrm{G}$, network operations typically represent $20 \%$ of the overall OPEX [4]. SONs have many facets: self-configuration, self-healing, and self-optimization (known collectively as "self- $*$ "). Of particular interest in this paper is the self-optimization of handover. Handover management is one of the use cases of the SON paradigm defined by the operators' alliance Next Generation Mobile Networks (NGMN) and is used to optimize handover performance between neighboring base stations, including femtocells.

Handover optimization in LTE has two key conflicting demands: minimizing the likelihood of dropped calls and minimizing unnecessary handovers. Two tunable parameters that govern handover performance are defined: the time to trigger (TTT) and the handover hysteresis value (Hys). Handover to a candidate base station can be only executed if that candidate base station has superior signal strength to that of the serving base station by an amount that is equal to or exceeding the Hys. Furthermore, this condition must remain true for a duration equal to the TTT. At the peripheries of neighboring 
macrocells, ping-pong handovers may occur as a result of the irregular boundaries of cells and the nonstationary nature of the channel. The LTE specifications [5] capture this possibility with the definition of the "handover-too-early" metric. The Hys and the TTT can be increased to reduce the likelihood of unnecessary handover; however, this will also increase the dropped call rate. This possibility is captured by the LTE specifications [5] with the definition of the "handover-too-late" metric. Thus, any algorithm tuning the Hys and the TTT must strike a delicate balance between unnecessary handovers and dropped call rates. The Hys and the TTT are intuitively obvious parameters to optimize macrocell handover but do not always fit well with indoor-outdoor handover. Consider the following two scenarios.

In the first scenario, as a mobile terminal approaches and passes through an external door, it will detect an increase in the reference signal received power (RSRP) [6] from an externally located macrocell. As a consequence, a measurement report will be triggered (i.e., by event type $A 3$ [7]) and transmitted from the mobile terminal to the femtocell base station, informing the femtocell that another base station has been detected and is a candidate for handover. The femtocell will use the measurement report to initiate handover to the macrocell if required. If the call is dropped or an unnecessary handover occurs, the tunable parameters will be modified accordingly.

In the second scenario, a mobile user approaches a window with low penetration loss. The increase in RSRP from the macrocell will cause the measurement report to be sent from the mobile user to the femtocell, potentially invoking handover. However, as the mobile user moves past the window, the relatively high received signal level from the macrocell will rapidly decline and trigger another measurement report from the mobile user to the macrocell, indicating that a superior signal can be obtained from the femtocell. Such actions will invoke a second handover, in quick succession, from the macrocell back to the femtocell (so-called ping-pong handover). Clearly, the second scenario illustrates where unnecessary handovers can potentially occur. Unnecessary handovers may have negative consequences for future handover performance since they will cause an increase in the Hys and TTT parameters and, in doing so, make future handover more conservative. Modifying the parameters in this fashion may be disadvantageous when the mobile user leaves the building as described in the first scenario. The handover response may now be so conservative that the call will be dropped before handover is executed. Note that there are occasions whereby an active mobile terminal approaches and pauses by a large window. Under such a circumstance, any handover to the macrocell base station is likely to be unnecessary; nonetheless, it would be preferable to avoid such an eventuality to ensure that closed subscriber group traffic remains assigned to the femtocell where possible.

The femtocell cannot differentiate between a terminal approaching a window or a door; therefore, attempts to tune the handover parameters without distinguishing between these scenarios will result in suboptimal performance. The best approach is to allow the parameters to be tuned and optimized in regions where handover is likely to be required (i.e., around external doors) but remain unchanged in areas where unnecessary handovers are likely to occur (i.e., around windows), i.e., to suppress handover requests in regions that have a history of unnecessary handovers. Unnecessary handovers place additional demands on the network, such as unnecessary consumption of radio channels (random access channels) and fixed links and additional processing load in admission control, bearer setting, and path switching, and have the potential to degrade significantly the quality of service of ongoing connections [8].

The objective of the scheme presented in this paper is to identify indoor regions where handover to external base stations should be permitted and regions where handover should be prohibited. This paper is not concerned with the tuning of Hys and TTT per se, but rather with the determination of regions where such tuning should be permitted. The specific algorithm used for tuning these parameters is decoupled from the work presented here, and consequently, the approach suggested in this paper can be combined with other tuning algorithms. Three principal regions are defined.

1) Areas of macrocell low signal strength. In such regions, a measurement report will not be generated. Such areas can be regarded as "null zones."

2) Areas of high signal strength from the macrocell where few unnecessary handovers occur. These regions are referred to as "permitted zones" since handover to the external base station will be beneficial. It is believed that these zones will coincide with architectural features such as external doors.

3) Areas of high signal strength from the macrocell where many unnecessary handovers occur. Such regions are referred to as "prohibited zones" since handover to the external base station should be suppressed because it is likely that a second handover (in the opposite direction) will soon follow. These regions will be consistent with architectural features such as windows and glass exterior walls. In practical systems, the number of times handover can be requested when the user moves into this area can be limited by means of the "reportamount" parameter [7]. The level of signaling required for each measurement report is minimal in comparison with each completed handover request (two sets of handovers are required for each handover ping-pong).

Crucially, TTT and Hys parameters apply to the entire cell. The problem under consideration is how to facilitate handover to the macrocell layer in a timely fashion while minimizing false positives, hence, minimizing unnecessary handovers. To this end, positional information is incorporated into this algorithm. The positional information at the point of a handover trigger can be derived (using the multiple antennas on the femtocell) from the angle of arrival (AoA) and RSRP of the user. For clarity, it should be noted that the positional information used in this algorithm is the location of regions within the radio environment where handover occurs and not the true physical location of the user. However, there may be a strong mapping between these two representations of location. This paper proposes a novel kernel self-organizing map (XSOM) algorithm and demonstrates an appropriate application of implementation within a femtocell environment for handover optimization. 


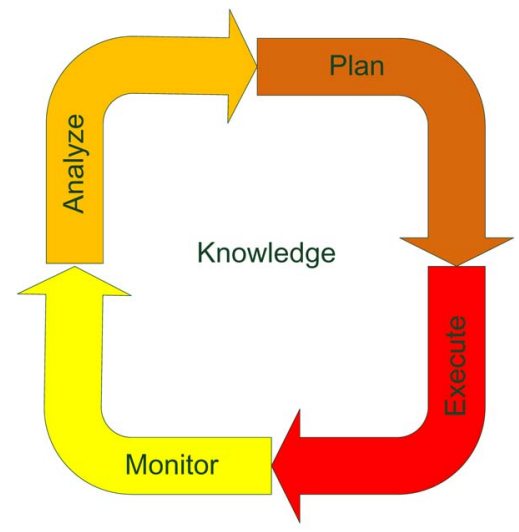

Managed Element

Fig. 1. Autonomic loop

This paper builds on the work previously described [9], where it was shown that a SOM can be used along with location detection in femtocells to optimize the handover process within a very large room. In the previous work, the system required an input from the user and was therefore not fully plug-and-play, as required by the SON paradigm. In this paper, the mechanism has been refined to realize a fully autonomous system that can detect the number of clusters required in the algorithm (by using the $X$-means algorithm). The novelty of the proposed scheme is the use of $X$-means within a neural networking algorithm demonstrating efficient handover optimization in an indoor environment equivalent to the size of an average room in a house.

The remainder of this paper is structured as follows. Section II provides a brief overview of autonomics and its use in this paper. Section III provides a brief explanation of the $\mathrm{XSOM}$ and the $X$-means algorithm used to inhibit handover. Section IV evaluates the performance of the proposed handover inhibition algorithm. Finally, Section V summarizes and concludes this paper.

\section{SElF-Organizing Networks}

The term autonomic computing refers to computing systems having the ability to self-manage and autonomically react to unpredictable events while hiding the complexity of the system to the end user. The inspiration for autonomic computing is the way that the nervous system regulates the operations of biological organisms [10]. This methodology was first applied by IBM in 2001 to handle the increasing complexity of managing computing systems with no external input. The autonomic paradigm is one in which time-consuming and error-prone tasks are undertaken by self-managing components, leaving human administrators free to concentrate on high-level policies. Autonomic systems are thus provided with high-level policies that are used to govern how the system will adapt and optimize to unforeseen changes. These policies state what the system should aim to do and not how it should be completed, which is the role of the autonomic element. Autonomic networks use the same paradigms created for autonomic computing systems but apply these ideas to network management [11]. The fundamental structure of autonomic systems is a control loop, which is represented in Fig. 1.
Fig. 1 shows the four stages involved in any autonomic system: monitoring, analyzing, planning, and execution. This is a useful structure for any self-optimizing system as the inherent feedback supports an autonomically adjusting management system. The four stages of the control loop constitute the fundamental building blocks of the autonomic system and are structured as follows.

- The monitoring phase is concerned with the acquisition, collation, and filtering of data concerning the managed element or its environment and constitutes the input to the autonomic system.

- The analyzing phase examines the data and determines potential actions to be taken to optimize the performance of the system or to take corrective action.

- The planning phase uses the conclusions of the analyzing phase to decide which specific actions should be taken to reconfigure or optimize the managed element.

- The execution phase translates the planned actions into a sequence of technology-specific commands.

This concept has been embraced by the NGMN and the $3 \mathrm{G}$ Partnership Project, and its principles provide the basis for the SON paradigm [3], [12]. This paradigm allows network operators to optimize continually their network by persistent reparameterizations of the dynamically changing network architecture and traffic loads. This functionality allows both macrocells (eNodeBs) and femtocells (HeNodeBs) to be deployed first and automatically self-manage themselves to meet the requirements of the network without human interaction. The work proposed in this paper considers self-optimization of handover [13]-[15].

The handover management use case, within SON, allows for the operation of procedures that result in improved handover performance and increased network capacity. Within the work described here, the autonomic system monitors when unnecessary handovers between the femtocell and the macrocell occur and seeks to reduce their number over time. However, the system should not prohibit seamless handover from the femtocell to the macrocell when genuinely required. A scheme that is too timid will lead to an increase in handover-too-late occurrences due to lack of responsiveness, and a scheme that is too aggressive will lead to an increase in handover-too-early occurrences.

To assess the performance of the algorithm, handover performance indicators (HPIs) are required. Appropriate HPIs are ping-pong handover ratio $\mathrm{HPI}_{\mathrm{pp}}$ and handover failure ratio $\mathrm{HPI}_{\mathrm{drop}}$, as given in the following [13]:

$$
\begin{aligned}
\mathrm{HPI}_{\mathrm{pp}} & =\frac{N_{\text {HPP }}}{N_{\text {Total }}} \\
\mathrm{HPI}_{\text {drop }} & =\frac{N_{\text {Hdrop }}}{N_{\text {Total }}} .
\end{aligned}
$$

Here, $N_{\mathrm{HPP}}$ is the number of handover ping-pongs, $N_{\mathrm{Hdrop}}$ is the number of failed handovers, and $N_{\text {Total }}$ is the total number of handovers. These metrics shown in (1) and (2) provide a measure of the performance of the algorithm and are usually expressed as a percentage. Ideally, the number of failed handovers and handover ping-pong occurrences would be 
zero (and, hence, so would $\mathrm{HPI}_{\mathrm{pp}}$ and $\mathrm{HPI}_{\mathrm{drop}}$ ). Unfortunately, practical systems are not ideal and do not operate optimally. In a practical system, handover ping-pong and handover failure will occur within the network, but the number of such occurrences can be reduced to minimize their effect.

Given the plug-and-play requirement for SON, it must be assumed that the user (or network provider) will not be in a position to provide any a priori knowledge concerning the radio environment within which the femtocell will be located. Such an assumption has a significant impact upon the design of any autonomous system because every radio environment is unique. Factors such as the placement of the femtocell, the architecture of the building (including building materials), the furniture in the building, and the number and location of external macrocell base stations all result in the topography of all null, permitted, and prohibited zones being unique for every femtocell. As a result, any algorithm used within femtocells for handover optimization must be able to configure and optimize fully itself to the environment in which it resides without any prior information on that environment. Consequently, oraclebased supervised learning strategies are not feasible; therefore, the type of learning used is restricted to unsupervised methods.

For a building of arbitrary shape and construction, an algorithm is required that can optimize handover performance with no operator input. To realize such an objective, the direction finding capability of MIMO systems is exploited to provide a profile of locations (or, more correctly, regions in the radio environment) where handover is genuinely required (permitted zones) and locations where unnecessary handovers are likely to occur (prohibited zones) by continually mapping regions where either successful or unnecessary handover has occurred and using this information to identify the permitted and prohibited zones. The XSOM is particularly useful in this context.

\section{Improved Kernel Self-Organizing Feature Map Using $X$-Means}

The purpose of the autonomic managed element, to be included within SON, is to optimize the handover process based on the application of an advanced kernel self-organizing (feature) map [16], [17]. The advancement is represented by the inclusion of $X$-means [18] within the XSOM; this is a novel adaptation. The monitoring phase of the SON algorithm is comprised of determining the location of the user by detecting where a measurement report has been triggered. The analyzing phase of the algorithm is based on an XSOM and allows the femtocell to learn the locations of the propagation environment that correspond to each type of zone. The planning phase then takes this information and determines an appropriate response, i.e., to permit or prohibit the requested handover. Finally, the execution phase translates the decision from the planning phase into LTE-specific commands and permits or prohibits the handover request.

The planning phase of the autonomic system uses an improved XSOM algorithm to provide the femtocell with a profile of locations in which handover may take place. A SOM is a type of an unsupervised neural network that allows for group learning to produce a low-dimensional output space from a high-dimensional discretized input space. The SOM is a form of an artificial neural network whose neurons are arranged in a 2-D lattice. The lattice can be regarded as a special case of a feedforward neural network with a single computation layer that is arranged into logical rows and columns. All neurons are connected to all inputs, and unlike other neural networks, the neurons have no activation function. Each input to the system is compared with all neurons, and the closest neuron is then used as the basis for the neurons to learn. These neurons can also hold information about previous handovers within the radio environment. The XSOM algorithm is a version of the SOM algorithm that nonlinearly transforms the data into a feature space. Once transformed, the distances between the weights and the inputs can be calculated nonlinearly.

The XSOM is particularly useful for detecting clusters within data, and in this paper, it is used to perform location fingerprinting based on received signal strength and AoA. The XSOM algorithm has an input space that is highly multidimensional, a weight space of the same dimension as the input and an output space with a smaller dimension than the input. An XSOM [19], [20] is a special version of the SOM that allows for a kernel method to replace the distance measurements within the SOM. Using kernel methods for a distance metric allows for a nonlinear mapping from the input space to a high-dimensional feature space, which results in additional detail (accuracy) at the point of interest and reduces the vector quantization error that is inherent to the SOM. Typically, there are four phases, which describe the learning process of the kernel SOM: initialization, competition, cooperation, and synaptic adaptation. The XSOM algorithm, on the other hand, augments the kernel SOM by adding a fifth stage, i.e., $X$-means. $X$-means allows for the SOM to be handled as a number of Voronoi cells.

\section{A. XSOM: Initialization}

The initialization of the SOM network presets the individual weight values of each neuron in the lattice to values drawn from a uniform distribution. The initial weight values will be distributed within the propagation region of the femtocell. Each input will be associated with a weight within the higher dimensional feature space. The input represents the geographical location obtained using the RSRP and the AoA from the mobile terminal at the time that the measurement report was generated.

\section{B. XSOM: Competition}

The next step of the process is for inputs to be applied to the system. Under operational conditions, this would occur every time a mobile terminal generates a measurement report (triggered by detection of a base station other than the serving base station). Since each input is connected to each neuron, the input and weight vectors have the same dimensions. The representation for an $a$-dimensional input and the weight vector associated with each neuron in the lattice is defined, respectively, as follows:

$$
\begin{aligned}
& \mathbf{x}=\left[x_{1}, x_{2}, \ldots, x_{a}\right]^{T}, \quad \mathbf{x} \in \mathbb{R}^{a} \\
& \mathbf{w}_{j}=\left[w_{j 1}, w_{j 2}, \ldots, w_{j a}\right]^{T}, \\
& j=1,2, \ldots, l ; \quad \mathbf{w}_{j} \in \mathbb{R}^{a} .
\end{aligned}
$$


Here, $l$ is the total number of weights in the network. Given that there is no activation function, the output of each neuron will be a combination of both the input and weight vectors. From a geometric perspective, the winning neuron in the XSOM is calculated based on Euclidean distance after implicitly transferring the map into a nonlinear feature space using the kernel trick [21]. The kernel trick is used because the nonlinear mapping of the input and weights allows for more detail at the point of interest, which reduces the vector quantization error of the system. The mapping of $\mathbf{x}$ to $\phi((x))$ can be implicitly accomplished with no knowledge of $\phi$. This means that only knowledge of the inputs, the weights, and kernel function $(K(\cdot, \cdot))$ are required. The competitive aspect of this algorithm is represented by the neuron whose weight vector provides the best match to the input vector will be selected as the winning neuron. If the index of the winning neuron is denoted by $i(\mathbf{x})$ within the lattice $\mathcal{L}$ (denoting the grid of neurons in the weight space), then the winner is given by

$$
i(\mathbf{x})=\arg \min _{j}\left\|\mathbf{x}-\mathbf{w}_{j}\right\|, \quad j \in \mathcal{L} .
$$

The distance can be written in terms of the kernel function, as shown in (6). The mapping to the feature space is completed using a kernel such that $K\left(\mathbf{x}_{i}, \mathbf{x}_{j}\right)=\phi\left(\mathbf{x}_{i}\right)^{T} \phi\left(\mathbf{x}_{j}\right)$, where $\phi(\mathbf{x})$ is the function that maps the data onto the feature space, i.e.,

$$
\begin{aligned}
\left\|\mathbf{x}-\mathbf{w}_{j}\right\|^{2} & =\left\|\phi(\mathbf{x})-\phi\left(\mathbf{w}_{j}\right)\right\|^{2} \\
& =K(\mathbf{x}, \mathbf{x})+K\left(\mathbf{w}_{j}, \mathbf{w}_{j}\right)-2 K\left(\mathbf{x}, \mathbf{w}_{j}\right)
\end{aligned}
$$

where a Gaussian kernel method is used as follows:

$$
K\left(\mathbf{x}, \mathbf{w}_{j}\right)=\exp \left(\frac{-\left\|\mathbf{x}-\mathbf{w}_{j}\right\|}{2 \sigma^{2}}\right) .
$$

Once the winner has been selected as the closest match to the input, it can be utilized by the next stage of the algorithm.

\section{X-Means}

$X$-means [18] is an advanced algorithm for cluster analysis that is based on the $k$-means algorithm. The disadvantage of $k$-means is that it requires the number of clusters, i.e., $k$, to be known in advance. $X$-means, however, does not suffer from this constraint. The ability of $X$-means to handle automatically an arbitrary number of clusters (i.e., an arbitrary number of prohibited and permitted zones) makes it particularly well suited to situations where no prior knowledge of the radio environment (and, hence, the number of clusters) is available. This removes the need for human intervention and is consistent with the requirement for plug-and-play functionality, as discussed in Section II. However, there is a requirement for the range that the number of clusters $k$ will fall within

$$
k_{\min } \leq k \leq k_{\max }
$$

$k_{\min }$ to $k_{\max }$ represents the range of $k$, which will be calculated using this algorithm and can be a default set of values for every femtocell. The inclusion of $X$-means within the XSOM allows for faster convergence times as a consequence of a reduction in the level of false learning within the system due to only updating the weights within the network that are within the region of the input. $X$-means operates after each iteration of the $k$-means algorithm by making local decisions about whether to split each Voronoi cell in half, to better fit the data. This allows the algorithm to start by using $k_{\text {min }}$, with increments as required, and to finish by using any value within the range [shown in (8)] that best fits the data.

1) The partitioning is completed by initially allocating $k_{\min }$ centroids randomly within the area of the network.

2) Each weight can be then allocated to its nearest centroid using

$q(\mathbf{w})=\arg \max _{c}\left\|\mathbf{w}_{j}-\mathbf{m}_{c}\right\|, \quad j \in \mathcal{L} ; \quad c \in[0, k]$

where $m$ denotes the centroid, $c$ denotes the index, and $q(\mathbf{w})$ denotes the index of the winning centroid. This results in the generation of Voronoi cells.

3) Now that each weight has been allocated to its corresponding centroid, the centroid must be updated using

$$
\mathbf{m}_{c}=\frac{1}{n} \sum_{j=1}^{n} \mathbf{w}_{j} .
$$

Each new centroid location $\mathbf{m}_{c}$ is the mean value of all the allocated weights. $n$ is the number of weights allocated to mean $c$.

4) Steps 2 and 3 are repeated until convergence of the centroid and allocated weights has been achieved.

5) Now that the weights within the network have been successfully allocated to their nearest centroids and the centroids have been accurately calculated, the number of centroids can be updated. The algorithm works by splitting each of the centroids into two centroids. Determining whether this split is valid is facilitated by the Bayesian information criterion (BIC). The BIC scoring operates by using posterior probabilities to score the models. To approximate the posteriors, up to normalization, we use

$$
\operatorname{BIC}\left(M_{s}\right)=\hat{l}_{s}(D)-\frac{p_{s}}{2} \cdot \log R .
$$

Here, $\hat{l}_{s}(D)$ is the log likelihood of the data taken at the maximum-likelihood point, $p_{s}$ is the number of parameters in $M_{s}$, and $R$ is the number of weights in data set $D$. The maximum-likelihood estimate for the variance is calculated using

$$
\hat{\sigma}^{2}=\frac{1}{R-k} \sum_{i}\left(\mathbf{x}_{i}-\mathbf{m}_{q(\mathbf{w})}\right)^{2}
$$

where $k$ is the current number of centroids being used in the $X$-means algorithm, and $i$ is the input index. The log likelihood of the data points that belong to centroid $m_{c}\left(\hat{l}_{s}\left(D_{c}\right)\right)$ and that include the maximum-likelihood estimates yields

$$
\begin{aligned}
\hat{l}_{s}\left(D_{c}\right)=-\frac{R_{c}}{2} \log (2 \pi)-\frac{R_{c} \cdot M}{2} \log \left(\hat{\sigma}^{2}\right) & \\
& -\frac{R_{c}-k}{2}+R_{c} \log R_{c}-R_{c} \log R .
\end{aligned}
$$


Within this equation, $R_{c}$ is the number of weights allocated to $m_{c}$. The number of parameters $p_{s}$ is the sum of $k-1$-class probabilities, $M \cdot k$ centroid coordinates, and one variance estimate, as shown in the following:

$$
p_{s}=(k-1)+(M \cdot k)+k .
$$

The number of clusters $k$ is increased based on the resultant BIC score until either the solution has converged or the condition stated in (8) is violated. Convergence is validated by comparing the BIC score of the final network to the BIC score of the initial solution.

\section{XSOM: Cooperation}

Once the winner for a given input vector has been selected and the weight has been assigned to its closest centroid, the weights of the neurons within the winner's sphere of influence are updated if they are linked to the same centroid as the winner. This constitutes a cooperative learning process since, unlike other competitive learning strategies, it is not just the winning neuron that has its weight values modified. This group learning strategy permits the network to converge more rapidly and accurately compared with the case where only the winner would modify its weights.

The sphere of influence is governed by a neighborhood function that determines how many of the winner's neighbors can undergo learning, as well as the degree to which they will learn. Within the sphere of influence, neighbors closer to the winning neuron will have their weights updated by a greater amount than those located further away. To achieve this, a distance metric between neurons in the lattice is required, where the distance between two neurons $e$ and $f$ is given by [similar to (6)]

$$
d_{f, e}=\left\|\phi\left(\mathbf{r}_{f}\right)-\phi\left(\mathbf{r}_{e}\right)\right\|^{2} .
$$

Here, $\mathbf{r}_{e}$ and $\mathbf{r}_{f}$ are the locations of neurons $e$ and $f$ in the lattice, respectively.

The neighborhood function should monotonically decay with distance from the winner. Furthermore, it should be at maximum at the winner $\left(d_{f, e}=0\right)$ and decay to zero as $d_{f, e} \rightarrow \infty$. A popular choice for the neighborhood function that satisfies these requirements is the Gaussian function

$$
h_{f, e}=\exp \left(-\frac{d_{f, e}^{2}}{2 \sigma^{2}}\right), f \in \mathcal{L}
$$

and this function is adopted in this paper.

The parameter $\sigma$ defines the width of the Gaussian function, and in essence, $\sigma$ determines the size of the sphere of influence around the winning neuron. When using an XSOM, the size of the sphere of influence (i.e., $\sigma$ ) is reduced over time; in practice, this translates to the number of iterations $n$. The width of the neighborhood function can be made to decay with time by making $\sigma$ decay with time. Here, this is realized by assigning an exponential decay to $\sigma$ as follows:

$$
\sigma(n)=\sigma_{0} \exp \left(-\frac{n}{\tau_{1}}\right)
$$

where $n$ is the iteration number, $\sigma_{0}$ is the initial value, and $\tau_{1}$ is a temporal decay time constant chosen by the designer. The rate of decay effectively defines the learning rate of the algorithm: The faster the learning rate is, the more quickly an optimal solution would be found. However, this limits the adaption of the algorithm. By incorporating temporal decay, (16) can now be rewritten as

$$
h_{f, e}(n)=\exp \left(-\frac{d_{f, e}^{2}}{2 \sigma^{2}(n)}\right), j \in \mathcal{L} .
$$

\section{E. XSOM: Synaptic Adaptation}

The adaption process is concerned with the execution of the weight update procedure for all neurons within the sphere of influence of the winner. This involves utilizing not only the sphere of influence but also the learning rate. Unfortunately, standard Hebbian learning is not suitable for this type of a network because repeated reinforcement (positive increase) of weights within a particular sphere of influence will cause the neurons contained therein to be driven to a state of saturation. Thus, a "forgetting" term is used to augment the update algorithm such that the strength of the weights is progressively decreased; the "forgetting" term is given by

$$
\mathbf{u}\left(y_{j}\right)=g\left(y_{j}\right) \mathbf{w}_{j} .
$$

Generally, $g\left(y_{j}\right)$ is a positive scalar function of neuron $j$ 's output. An appropriate choice for this function is given by

$$
g\left(y_{j}\right)=\eta y_{j} .
$$

Parameter $\eta$ is the learning rate. In practice, the learning rate also decays with time (or iterations); therefore, it is an exponentially decreasing function, as shown in the following:

$$
\eta(n)=\eta_{0} \exp \left(-\frac{n}{\tau_{2}}\right)
$$

where $\eta_{0}$ is the initial value, and $\tau_{2}$ is the second time constant. The augmented Hebbian weight update equation can be written as follows:

$$
\Delta \mathbf{w}_{j}=\eta y_{j} \mathbf{x}-\eta y_{j} \mathbf{w}_{j} .
$$

By setting $y_{j}=h_{j, i(\mathbf{x})}$, the weight update equation can be written as follows:

$$
\Delta \mathbf{w}_{j}=\eta h_{j, i(\mathbf{x})}\left(\mathbf{x}-\mathbf{w}_{j}\right) .
$$

Thus, the weights for neuron $j$ within the sphere of influence of the winner are iteratively updated according to the rule given by

$$
\mathbf{w}_{j}(n+1)=\mathbf{w}_{j}(n)+\eta(n) h_{j, i(\mathbf{x})}(n)\left(\mathbf{x}(n)-\mathbf{w}_{j}(n)\right) .
$$

When the neurons have been continuously updated over a period of time, the locations of the neurons will converge to optimal locations as the learning rate has become low, and the neighborhood no longer updates nodes other than the winner. Once this happens, the locations of both permitted and prohibited zones have been identified. 
TABLE I

Simulation DETAILS

\begin{tabular}{|l|l|}
\hline Detail & Specifics \\
\hline Simulation dimensions & $7 \mathrm{~m} \times 9 \mathrm{~m}$ \\
Room dimensions & $7 \mathrm{~m} \times 7 \mathrm{~m}$ \\
Exit area & $2 \mathrm{~m} \times 7 \mathrm{~m}$ \\
No. of mobile terminals & 1 \\
Direction change time & $1.0 \mathrm{sec}$ \\
Movement speed & $1-3 \mathrm{~m} / \mathrm{s}$ \\
Initial position & center \\
Mobility model & random walk \\
Propagation model & single-slope \\
Error & 0m \\
\hline
\end{tabular}

\section{Simulation Model and Results}

To evaluate the effectiveness of the proposed neural networking algorithm, a simulation model was developed within the well-known simulator Network Simulator 3 (NS3). To test the performance of the XSOM, which is described in Section III, scenarios have been modeled that incorporate both prohibited zones and permitted zones within various room sizes. When a handover trigger is the input to the proposed algorithm, the system has detected that another base station yields a stronger signal (by a Hys) for a prescribed period of time (TTT). The handover trigger indicates that a handover has been requested.

The simulation model was used to determine the effectiveness of the proposed algorithm in identifying each type of zone. Once handover has been requested, a decision on whether to allow or inhibit this handover then takes place. As the modeled system operates using an event-based paradigm focusing on handover instances and takes a generic approach, the choice of propagation and mobility models only has a secondary effect upon the results. The choice of such models does not affect the generality of the outcome. Since indoor locations are inherently complex radio environments (due to clutter and other obstacles), position estimation error has also been investigated within the simulations; this does not significantly affect the learning rate or the accuracy of the system.

The requirement for brevity prohibits the discussion and analysis of extensive scenarios to demonstrate the generality of the algorithm. However, two specimen scenarios have been chosen to demonstrate key concepts and to illustrate typical performance. The first scenario incorporates one prohibited zone and one permitted zone. The second scenario incorporates two prohibited zones and a single permitted zone. The simulation details summarized in Table I are common to both case studies.

In these simulations, user mobility was represented using a modified random-walk mobility model. The random-walk mobility model allows for a random change of direction after a defined period of time (or distance traveled) and has been modified to mimic the likely movement of users at both prohibition and prohibited zones. The model ensures that the mobile user exits the room when they move within a permitted zone (the user must leave through a door). When a user moves into the region of a prohibited zone, the user will maintain a constant velocity most of the time and will pause within the region otherwise. The modifications in the prohibited zone models a user that usually walks past, but occasionally stops at, a window. A single-slope propagation model has been also incorporated, which modifies the propagation characteristics that are perceived by the mobile terminal, depending on its location within the radio environment of the specific scenario being modeled. Within the propagation model, both the signal strengths of a single femtocell and a single macrocell are used to determine whether there is a requirement for handover. Once the terminal has informed the serving femtocell that another base station offers a signal that is stronger by a fixed Hys for a prescribed period of time (TTT), the femtocell base station will make a decision whether to allow or to prohibit handover using the mechanism already described.

Within an LTE system, when the RSRP of a base station other than the serving base station (detected by the mobile terminal) is higher than the serving base station by a Hys for the TTT period, a measurement report is generated. This measurement report then initiates the handover process and can be considered as a handover trigger. In an LTE system that utilizes the proposed algorithm, handover is then permitted or prohibited based on previous experience of handovers in the radio region where the handover trigger was generated. To facilitate such decisions, the regions of the propagation environment that relate to prohibited and permitted zones must first be determined from the regions of the radio environment where handover triggers occur. Each weight within the neural network has the ability to retain knowledge of previous handovers within that area. Specifically, the system will detect the regions within the radio environment where unnecessary handovers can occur and will seek to reduce them over time. By reducing the occurrences of unnecessary handovers within the femtocell environment, the number of handovers that occur has been improved.

Two case studies in which the mechanism has been implemented within will now be considered. The case studies demonstrate that the mechanism can effectively adapt to the number of permitted and prohibited zones that occur in an autonomic fashion. After the mechanism is evaluated for both case studies, the $X$-means algorithm and its ability to detect the number of clusters will be discussed.

\section{A. Case Studies}

The first case study presented here incorporates a single permitted zone and a single prohibited zone. The second case study incorporates a single permitted zone and two prohibited zones. Once the femtocell is initialized, the autonomic system begins to optimize the handover process by utilizing the control loop. The monitoring phase of the autonomic system detects the locations of handover triggers. Once the location has been detected, the analyzing phase determines whether the handover trigger is within a permitted or prohibited zone. The planning phase then allows the system to decide on an appropriate response (either to permit or to prohibit the handover trigger). The location and the resulting status of each trigger are shown in Figs. 2-5.

At initialization, the proposed handover optimization algorithm is conservative and allows all handovers to be permitted everywhere, as shown in Figs. 2 and 3. Once the system has commenced learning the regions of the propagation environment where handover is not required, handover can be solely prohibited within the prohibited zone and exclusively permitted within the permitted zone, as shown in Figs. 4 and 5. 


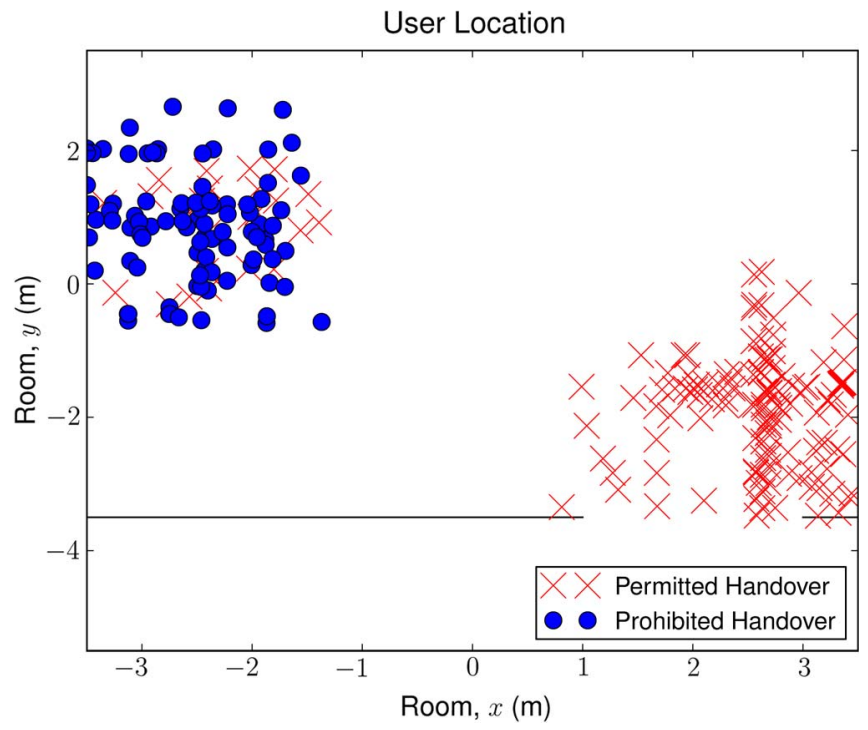

Fig. 2. Case study 1: Suppressions 1-100.

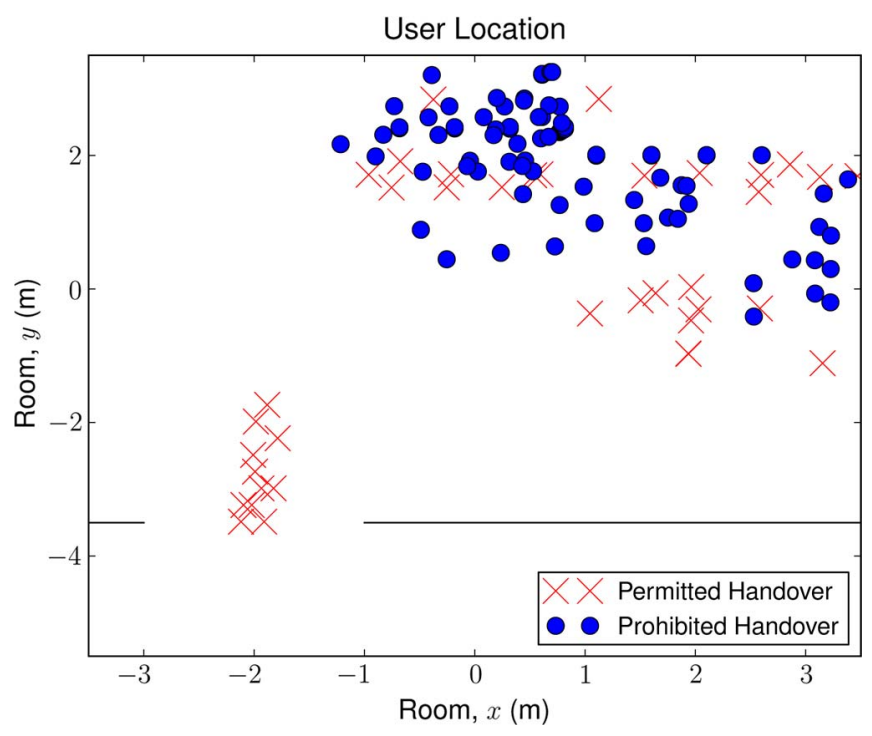

Fig. 3. Case study 2: Suppressions 1-100.

It should be noted that the handover triggers occur around the regions where the macrocell RSRP is greater than the femtocell RSRP; this can be either a macrocell-to-femtocell handover or a femtocell-to-macrocell handover.

In a practical environment, the position of the user would incorporate a positional error due to indoor environments being inherently complex radio environments. The location error within the indoor environment is due to the effects of clutter and other obstacles. The well-known robustness of neural networks to noise is demonstrated by the algorithm being relatively insensitive to positional accuracy (a range of positional error with uniform distribution is examined). The movement of the neurons (due to error) during learning will, in effect, cancel each other out. The learning curve of the system for a range of estimation error is shown in Figs. 6 and 7.

When the femtocell is initialized, the algorithm's learning curve, as shown in Figs. 6 and 7, is conservative; therefore, the system is nonrestrictive and allows all handovers to occur. As

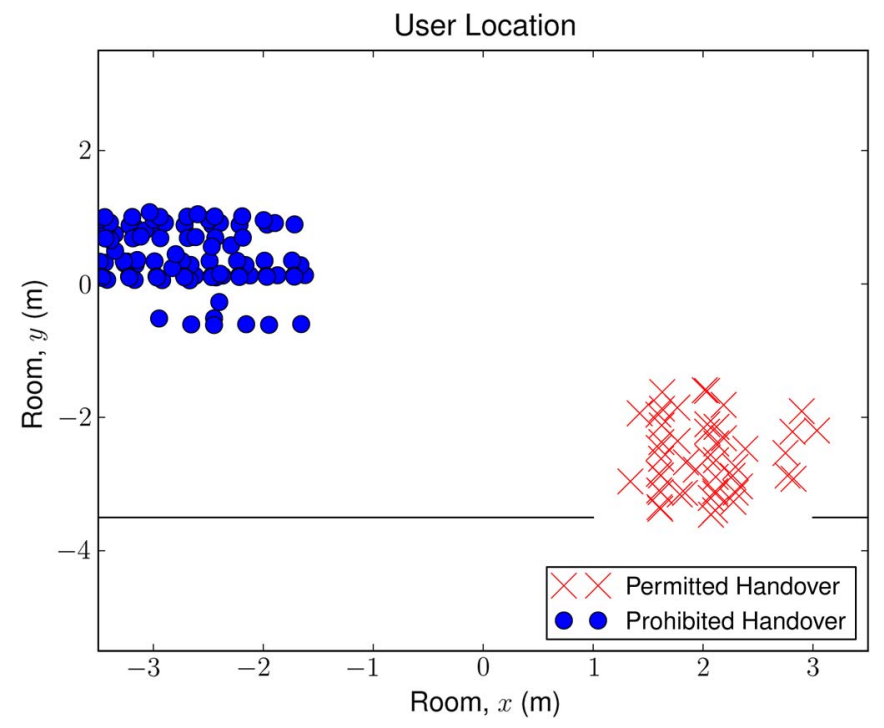

Fig. 4. Case study 1: Suppressions 301-400.

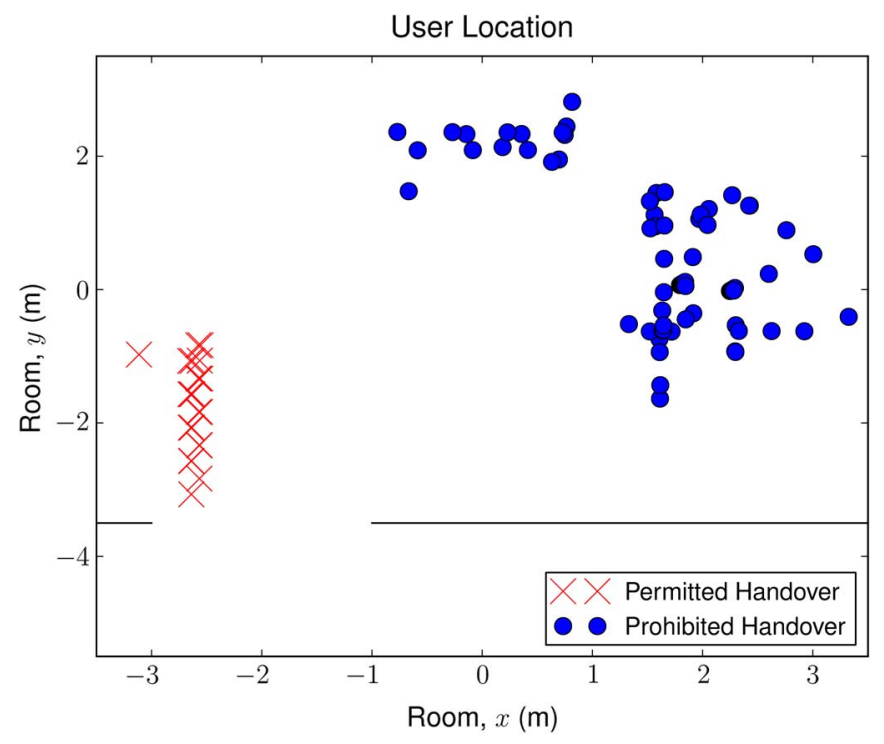

Fig. 5. Case study 2: Suppressions 301-400.

the algorithm gradually learns from experience of unnecessary handovers, it prohibits handovers within the regions of prohibited zones while still permitting handover within the region of permitted zones. This is apparent by comparing Fig. 2 or 3 with Fig. 4 or 5, respectively. It can be seen that, once the mechanism has been given time to learn the environment in which it has been deployed, handover will exclusively occur within the permitted zones and exclusively prohibited within the prohibited zones. However, there is a tradeoff between rapid learning of the environment (leading to performance improvement) and adaptability. Once the algorithm has fully adapted to a radio environment, changes in that radio environment (e.g., changes in the location of furniture and other reflectors) will lead to suboptimal performance. This eventuality can be overcome by a simple manual reset.

The optimization algorithm can be compared with the standard LTE system based on HPIs. The handover ping-pong ratios for these specific scenarios are shown in Figs. 8 and 9. 


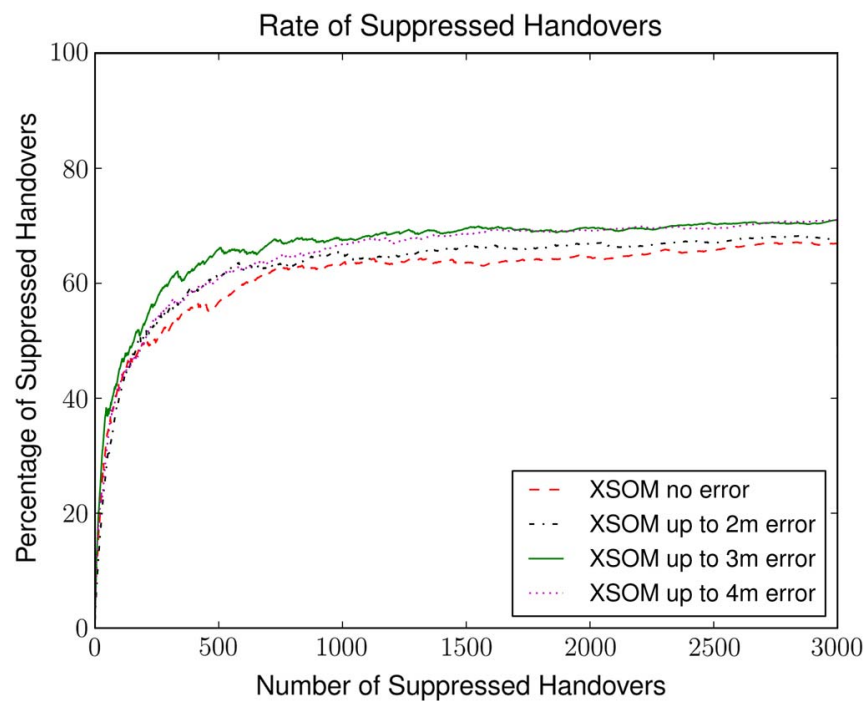

Fig. 6. Case study 1: Handover suppression rate with and without error.

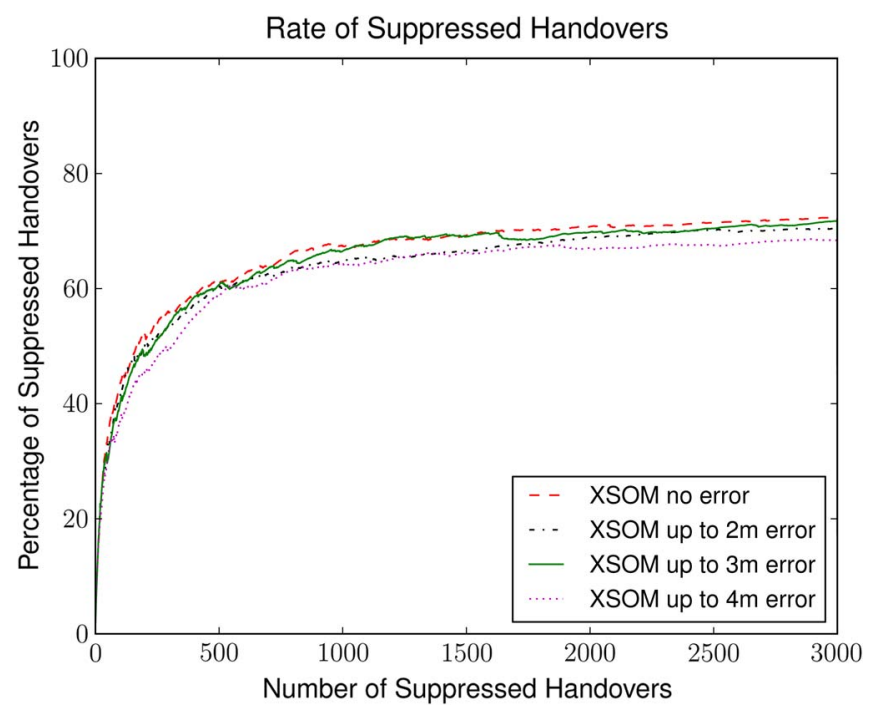

Fig. 7. Case study 2: Handover suppression rate with and without error.

The handover dropped call ratios for these specific scenarios are shown in Figs. 10 and 11. The HPI figures include the performance of the network with and without the algorithm being proposed.

As shown in Fig. 8 and 9, the situation includes a high level of handover ping-pong occurrences. The standard LTE system is shown to converge rapidly at a high level of handover pingpongs. However, when the proposed algorithm is included, the number of handover ping-pongs is significantly reduced and would continually reduce as the system learns the details about the environment.

The level of dropped calls within the system for both the modified and unmodified LTE systems for both case studies is shown in Figs. 10 and 11. The values within this graph follow (2) and are therefore the number of dropped calls divided by the total number of handovers at that point in time. In the modified system, the number of dropped calls is lower than that with the standard LTE system.

As the learning algorithm is of an unsupervised nature, the algorithm is nonrestrictive at initialization to allow all handovers

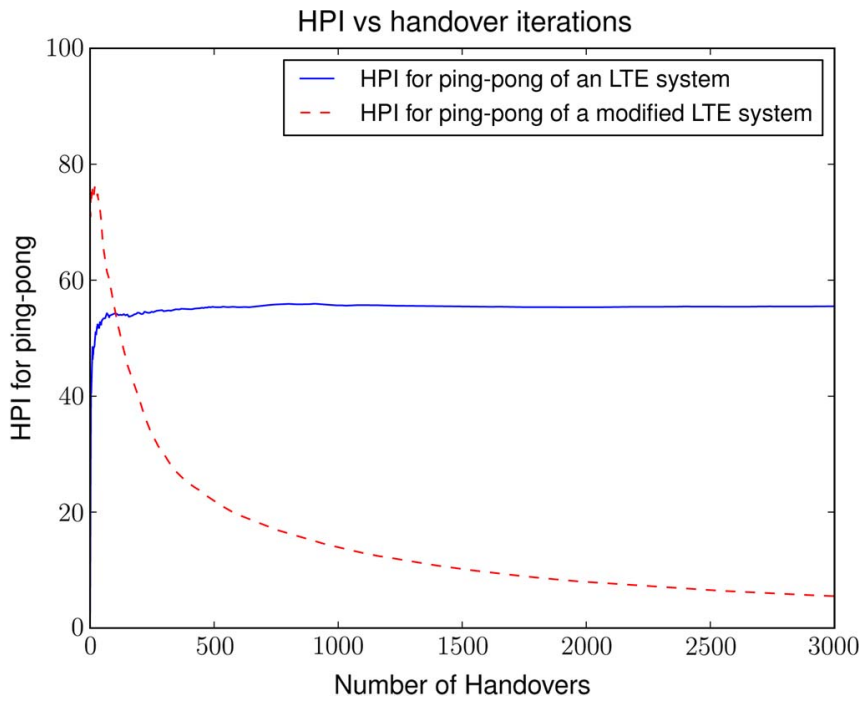

Fig. 8. Case study 1: Rate of ping-pong handovers.

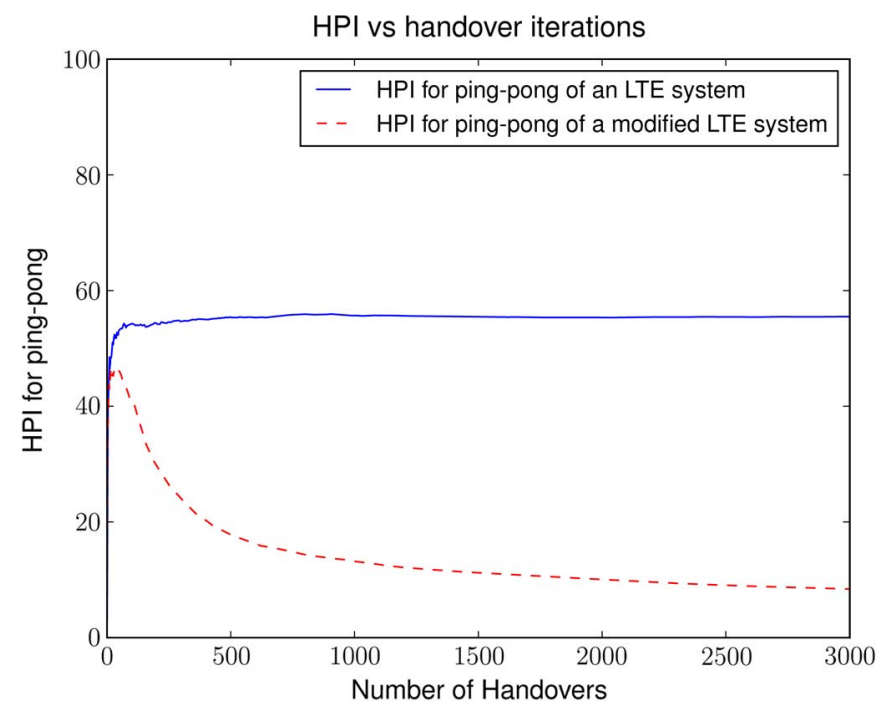

Fig. 9. Case study 2: Rate of ping-pong handovers.

to occur. After the femtocell has been set up, there is then a learning curve involved to prohibit progressively unnecessary handovers, as shown in Figs. 12 and 13. An initial value for unnecessary handovers is used for each neuron in the network, and scalar reinforcement is carried out, which eventually indicates whether the terminal state is a state that should be avoided (prohibited zone). As can be shown by comparing both the Kohonen SOM algorithm and the improved XSOM algorithm shown in Figs. 12 and 13, the XSOM provides an improved performance because its convergence time is faster compared with the Kohonen SOM. The improved performance of the algorithm is due to the change in distance metric (now kernel trick and not Euclidean distance) and the addition of $X$-means into the weight updating process. Figs. 12 and 13 show that, as the mobile terminal moves around the indoor radio environment, the number of prohibited handovers converge to about $70 \%$. These figures were generated using 30 simulation runs to provide an ensemble average. The level of prohibited handovers and the resulting convergence rate is linked to the accuracy of 


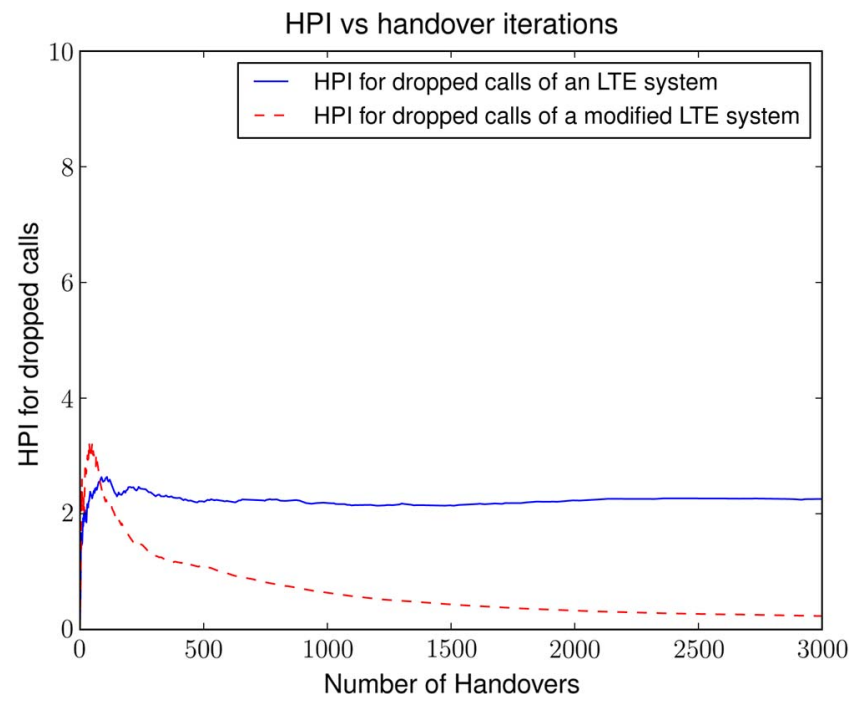

Fig. 10. Case study 1: Rate of dropped calls.

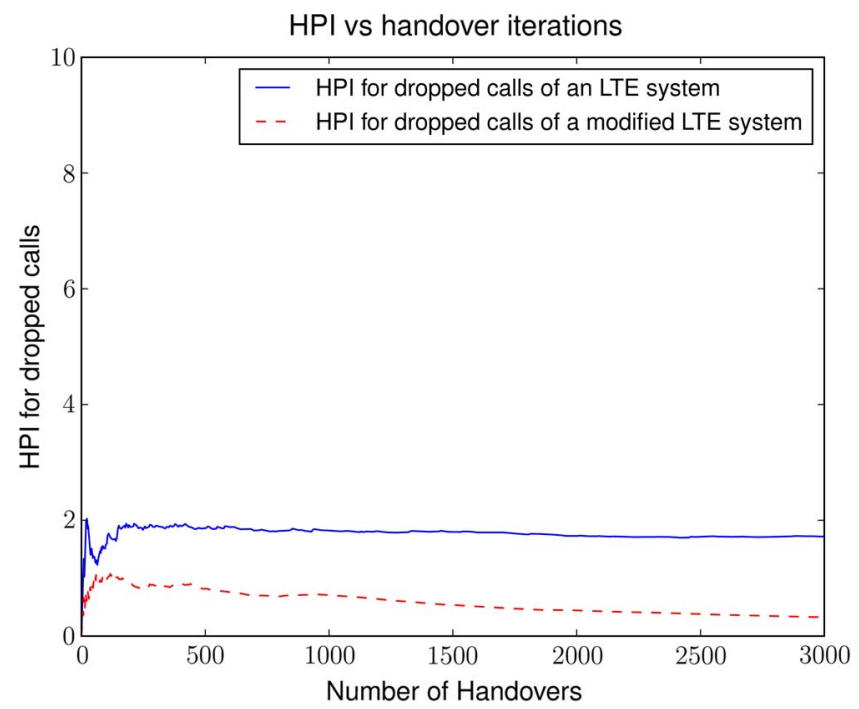

Fig. 11. Case study 2: Rate of dropped calls.

the $X$-means algorithm and its ability to detect effectively the correct number of clusters (as discussed in Section IV-B).

\section{B. X-Means Cluster Detection}

The addition of $X$-means to the kernel SOM algorithm improves the performance of a simple kernel SOM algorithm by splitting the neurons into the appropriate number of Voronoi cells, i.e., three in this case. The number of Voronoi cells $k$ directly correlates with the number of windows and doors in the indoor area. $X$-means has the ability to calculate autonomically the number of clusters required in the system. Fig. 14 shows the average number of clusters calculated using $X$-means with different numbers of data points and compares this with the ideal number of clusters.

When using a nonideal value for $k$, the proposed system will still yields an overall improvement over the unmodified LTE system, but the convergence rate may be suboptimum. Fig. 15 compares different values of $k$ for different numbers of handovers suppressed. When $k$ is similar to the correct number

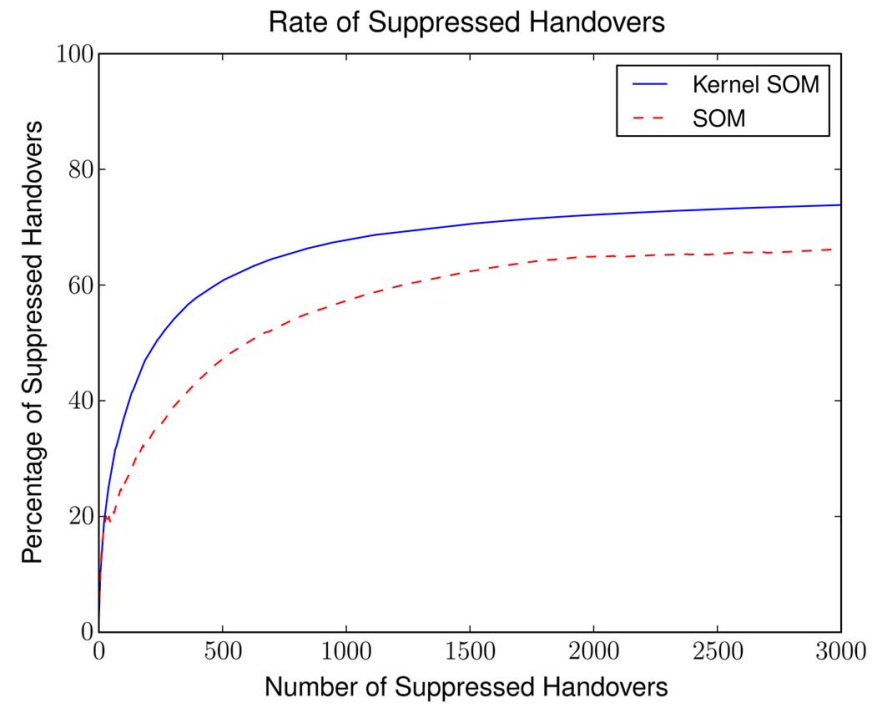

Fig. 12. Case study 1: Handover suppression rate of the SOM and the advanced XSOM.

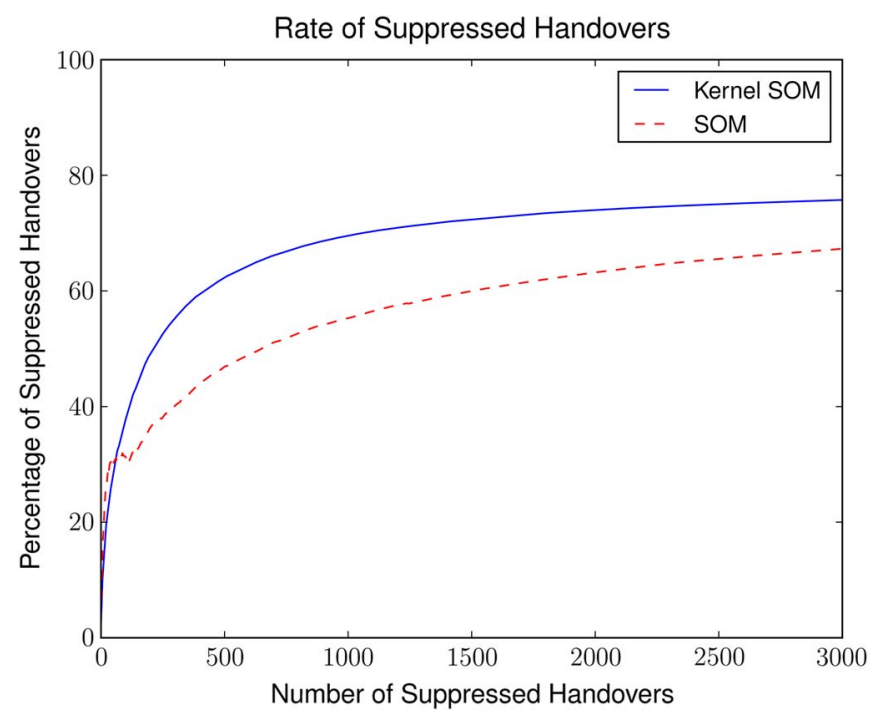

Fig. 13. Case study 2: Handover suppression rate of the SOM and the advanced XSOM.

of clusters, the overall performance is not significantly different, and the percentage of suppressed handovers converges close to a common value. However, when the value for $k$ is dramatically wrong (e.g., when $k$ is 8 or 10 ), the performance of the algorithm is impacted. Thus, the use of $X$-means is valid within plug-and-play functionality of SON within the indoor environment, due to it being able to estimate autonomously how many handover areas there are within the system.

The case studies show that a considerable number of handover ping-pongs occur within the network. The locations of these ping-pongs have been detected, and the handovers within this region have been increasingly prohibited. Once a handover trigger has been paired to its closest neuron, learning can occur to optimize handover performance. $X$-means (see Section III) is used to aid the process of the XSOM algorithm (see Section III) to increase the rate of convergence, which reduces the learning time of the system. It should be noted that the system rapidly starts to reduce the number of handovers, and any reduction 


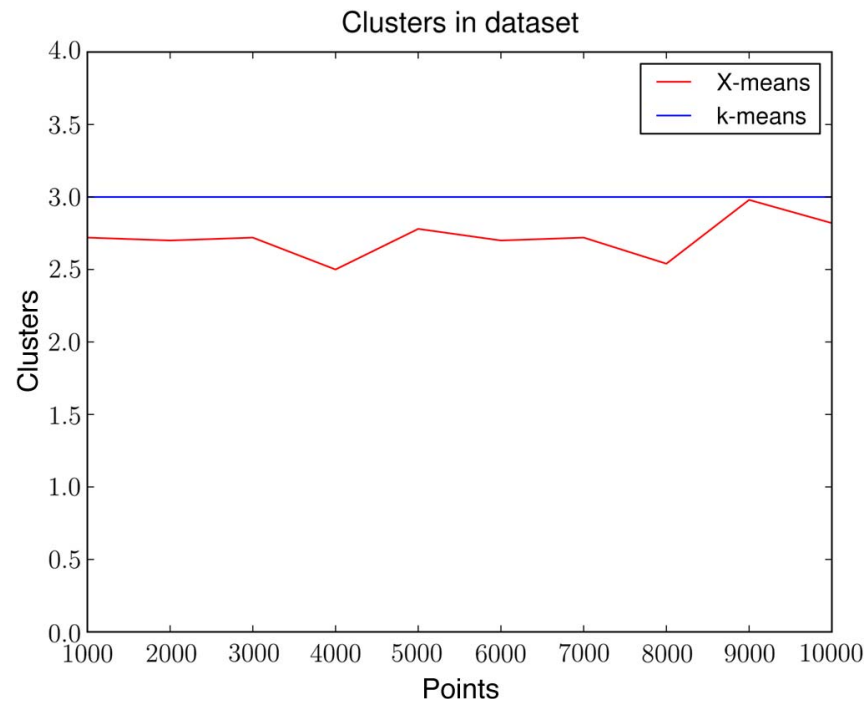

Fig. 14. $X$-means calculating $k$ with different numbers of data points.

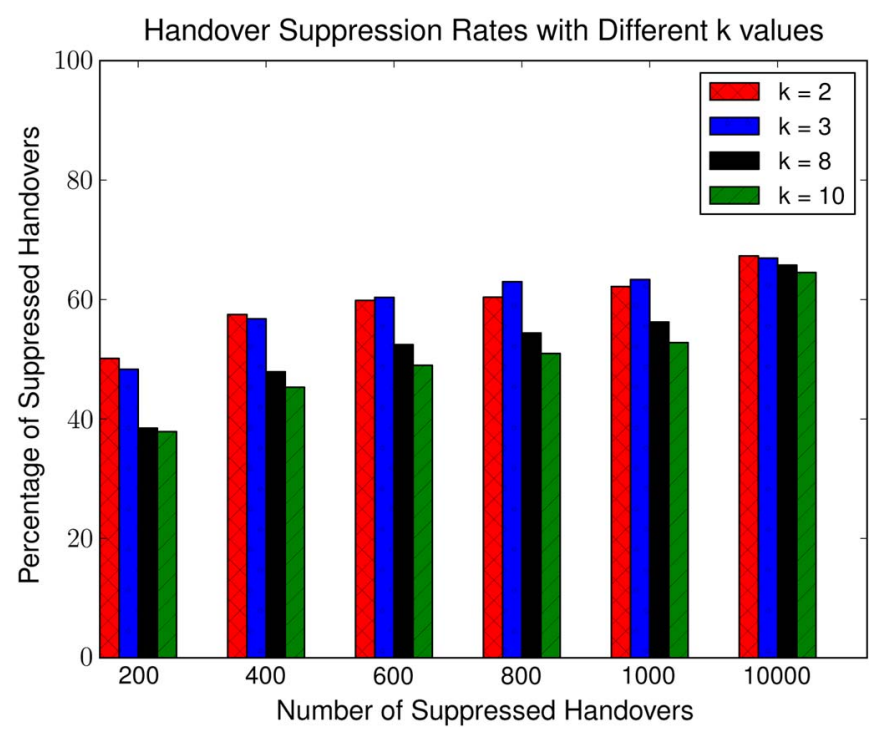

Fig. 15. Handover suppression rate with different $k$ values.

in the number of handovers represents an improvement in system performance quality. The reduction of handovers of up to $70 \%$, in effect, increases the overall capacity of the system by improving the efficiency of the handover performance. It should be noted that the algorithm is not given any prior information regarding the location of the windows, doors, or where handovers will occur. This information is gained via unsupervised learning. By modifying the handover process to include the option of permitting or prohibiting handovers, using the improved XSOM algorithm, the handover process becomes considerably more efficient.

\section{Summary AND CONCLUSION}

This paper has proposed a machine learning algorithm to improve handover performance between indoor femtocells and external macrocells for LTE. Based on experience, the algorithm builds a representation of the radio environment and seeks to suppress handover in regions where unnecessary handover has been executed previously. The algorithm, which runs on the femtocell base station, requires no prior knowledge of the architecture of the building in which it is deployed; thus, it is fully consistent with the SON plug-and-play requirement. It is demonstrated that the algorithm has the ability to reduce unnecessary handover by up to $70 \%$. Further work will consider optimal tuning of the TTT and handover Hys parameters in situations where handover is deemed necessary. As part of this investigation, the effects of external interference and the impact on macrocellular load will be considered to support analysis of call drop rates (handover too late) and other quality metrics.

\section{ACKNOWLEDGMENT}

The authors would like to thank D. Pelleg and A. Moore from the School of Computer Science, Carnegie Mellon University, Pittsburgh, PA, USA, for their version of the $X$-means code and their help with implementation issues.

\section{REFERENCES}

[1] Technical Specification Group Services and System Aspects; Universal Mobile Telecommunications System (UMTS); Mobility procedures for Home Node B; Overall Description; Stage 2, Third-Gen. Partnership Project, Valbonne, France, 2009, 3GPP TS 25.367. V8.0.0.

[2] S. Brightfield, "Femtocells-How to get the most out of your network," 4G World Presentation, Oct. 2011.

[3] Technical Specification Group Services and System Aspects; Telecommunications Management; Self Organizing Networks; Concepts and Requirements (Rel. 8), Third-Gen. Partnership Project, Valbonne, France, Dec. 2008, 3GPP TS 32.500 V8.0.0.

[4] "Motorola LTE self organizing networks," Motorola, Schaumburg, IL, USA, Tech. Rep., 2009.

[5] Digital Cellular Telecommunications System (Phase 2+); UMTS; Telecommunications Management; SON; Policy NRM; IRP; Requirements, ETSI TS 132.521 V9.0.0, Apr. 2010.

[6] Technical Specification Group Radio Access Network; Evolved Universal Terrestrial Radio Access (E-UTRA); Physical Layer-Measurements (Rel. 8), Third-Gen. Partnership Project, Valbonne, France, Dec. 2008, 3GPP TS 36.214 V8.5.0.

[7] Technical Specification Group Radio Access Network; Evolved Universal Terrestrial Radio Access (E-UTRA); Radio Resource Control (RRC); Protocol Specification (Rel. 9), Third-Gen. Partnership Project, Valbonne, France, Jun. 2010, 3GPP TS 36.331 V9.3.

[8] J. Alonso-Rubio, "Self-optimization for handover oscillation control in LTE," in Proc. IEEE NOMS, Osaka, Japan, Apr. 2010, pp. 950-953.

[9] N. Sinclair, D. Harle, I. A. Glover, and R. C. Atkinson, "A kernel methods approach to reducing handover occurrences within LTE," in Proc. Eur. Wireless Conf., Apr. 2012, pp. 1-8.

[10] P. Horn, "Autonomic computing: IBM's perspective on the state of information technology," IBM Corp., Armonk, NY, USA, Tech. Rep., 2001.

[11] J. Strassner, "The role of autonomic networking in cognitive networks," in Cognitive Networks: Towards Self-Aware Networks, 1st ed. Hoboken, NJ, USA: Wiley, 2007, ch. 2, pp. 23-52.

[12] "The benefits of SON in LTE," Third-Gen. Partnership Project, Valbonne, France, Tech. Rep., 2009, White Paper.

[13] T. Jansen, I. Balan, J. Turk, I. Moerman, and T. K. Kürner, "Handover parameter optimization in LTE self-organizing networks," in Proc. IEEE 72nd Veh. Technol. Conf. Fall, Sep. 2010, pp. 1-5.

[14] K. Kitagawa, T. Komine, T. Yamamoto, and S. Konishi, "A handover optimization algorithm with mobility robustness for LTE systems," in Proc. IEEE 22nd Int. Symp.PIMRC, Sep. 2011, pp. 1647-1651.

[15] L. Ewe and H. Bakker, "Base station distributed handover optimization in LTE self-organizing networks," in Proc. IEEE 22nd Int. Symp. PIMRC, Sep. 2011, pp. 243-247.

[16] T. Kohonen, "Self-organized formation of topologically correct feature maps," Biol. Cybern., vol. 43, pp. 59-69, 1982.

[17] S. Haykin, "Self-organizing maps," in Neural Networks and Learning Machines, 3rd ed. Upper Saddle River, NJ, USA: Pearson, 2009, ch. 9, pp. 453-502. 
[18] D. Pelleg and A. W. Moore, "X-means: Extending k-means with efficient estimation of the number of clusters," in Proc. 17th Int. Conf. Mach. Learn., 2000, pp. 727-734.

[19] D. MacDonald and C. Fyfe, "The kernel self-organising map," in Proc. 4th Int. Conf. Knowl.-Based Intell. Eng. Syst. Allied Technol., 2000, pp. 317-320.

[20] M. Martín-Merino and A. Munoz, "Extending the SOM algorithm to nonEuclidean distances via the kernel trick," in Proc. Neural Inf. Process., 2004, pp. 150-157.

[21] B. Schölkopf, "The kernel trick for distances," Cambridge, U.K., TR MSR 2000-51, 1993.

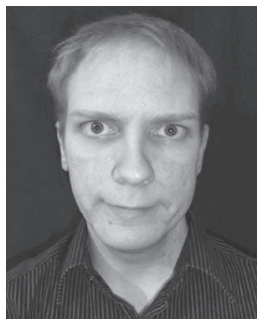

Neil Sinclair (S'09) received the Master's degree in electronic and digital systems from the University of Strathclyde, Glasgow, U.K., in 2009. He is currently working toward the Ph.D. degree with the Center for Intelligent and Dynamic Communications, Department of Electronic and Electrical Engineering, University of Strathclyde.

His research interests include neural networks and their application to handover management within Long-Term Evolution networks.

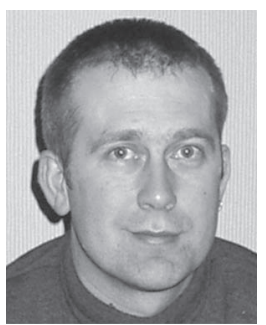

David Harle (M'90) received the B.Sc.(Hons.) degree in electronic and electrical engineering and the Ph.D. degree in telecommunications engineering from the University of Strathclyde, Glasgow, U.K., in 1984 and 1990, respectively.

$\mathrm{He}$ is currently a Senior Lecturer with the Broadband Optical Networks Group, Department of Electronic and Electrical Engineering, University of Strathclyde, Glasgow, U.K. He is the author of over 100 international conference and journal publications, one technical textbook, and three patents and undergraduate texts. His research interests include performance evaluation, design, and management issues associated with current and future wireless, broadband, and optical communication systems; network survivability; and the operation of routing protocols in multiservice environments.

Dr. Harle is a member of The Institution of Engineering and Technology and is a Chartered Engineer in U.K.

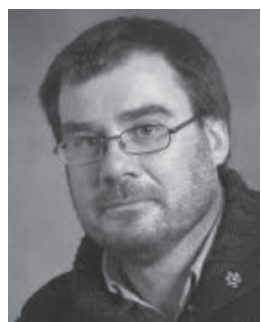

Ian A. Glover (M'90) received the B.Eng. degree in electronic and electrical engineering and the $\mathrm{Ph} . \mathrm{D}$. degree in radio science from the University of Bradford, Yorkshire, U.K., in 1981 and 1987, respectively.

$\mathrm{He}$ is currently a Professor of radio physics and wireless system engineering and a Department Head with the Department of Engineering and Technology, University of Huddersfield, Huddersfield, U.K. He is also a Visiting Professor of radio science with the Federal University of Campina Grande, Campina Grande, Brazil. His main research interests include the application of radiometric and wireless communication methods to insulation condition monitoring and asset management of high-voltage plants in future smart grids.

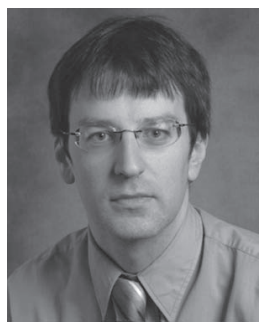

James Irvine (M'96-SM'01) received the B.Eng. (Hons.) degree in electronic and electrical engineering and the Ph.D. degree in coding theory from the University of Strathclyde, Glasgow, U.K., in 1989 and 1994, respectively.

He is currently a Reader with the Department of Electronic and Electrical Engineering, University of Strathclyde, Glasgow, U.K., where he also leads the Mobile Communications Group. He is a coauthor of seven patents and the books Digital Mobile Communications and the TETRA System (Wiley, 1999) and Data Communications and Networks: An Engineering Approach (Wiley, 2006). His research interests include mobile communication and security, particularly resource allocation and coding theory. Dr. Irvine is a member of The Institution of Engineering and Technology, a Fellow of the Higher Education Academy, and is a Chartered Engineer.

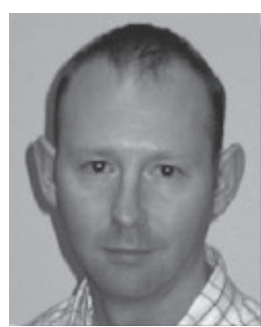

Robert C. Atkinson (M'03-S'98-SM'07) received the B.Eng. (Hons.) degree in electronic and electrical engineering; the M.Sc. degree in communications, control, and digital signal processing; and the Ph.D. degree in mobile communications systems from the University of Strathclyde, Glasgow, U.K., in 1993, 1995, and 2003, respectively.

His research interests include communications systems utilizing applied machine learning and optimization theory, such as autonomic management of fourth-generation mobile systems, intelligent radio access network selection, and wireless channel characterization.

Dr. Atkinson is a member of The Institution of Engineering and Technology. 